\title{
Relation of Serum Phosphorus Levels to the Incidence of Atrial Fibrillation (from the Atherosclerosis Risk In Communities [ARIC] Study)
}

\author{
Faye L. Lopez, MS, MPH ${ }^{a,}{ }^{,}$, Sunil K. Agarwal, MD, PhD ${ }^{b}$, Morgan E. Grams, MD, MHS ${ }^{\mathrm{c}}$, \\ Laura R. Loehr, MD, PhD ${ }^{b}$, Elsayed Z. Soliman, MD, MSc, MS ${ }^{d}$, Pamela L. Lutsey, PhD, \\ MPH $^{a}$, Lin Y. Chen, MBBS, MS ${ }^{e}$, Rachel R. Huxley, DPhil ${ }^{a}$, and Alvaro Alonso, MD, PhDa \\ aDivision of Epidemiology and Community Health, University of Minnesota School of Public \\ Health, Minneapolis, Minnesota ${ }^{b}$ Department of Epidemiology, University of North Carolina \\ Gillings School of Global Public Health, Chapel Hill, North Carolina 'Department of Epidemiology, \\ Johns Hopkins Bloomberg School of Public Health, Baltimore, Maryland dDivision of Public Health \\ Sciences, Department of Epidemiology, Wake Forest University School of Medicine, Winston- \\ Salem, North Carolina ${ }^{\mathrm{e}}$ Cardiovascular Division, University of Minnesota Medical School, \\ Minneapolis, Minnesota
}

\begin{abstract}
High serum phosphorus levels have been linked with vascular calcification and greater cardiovascular morbidity and mortality. We assessed whether serum phosphorus was associated with the atrial fibrillation (AF) incidence in a large community-based cohort in the United States. Our analysis included 14,675 participants (25\% black, 45\% men) free of AF at baseline (1987 to 1989 ) and with measurements of fasting serum phosphorus from the Atherosclerosis Risk In Communities (ARIC) study. The incidence of AF was ascertained through the end of 2008 from study visit electrocardiograms, hospitalizations, and death certificates. Cox proportional hazard models were used to estimate the hazard ratios of AF by the serum phosphorus levels, adjusting for potential confounders. During a median follow-up of 19.7 years, we identified 1,656 incident $\mathrm{AF}$ cases. Greater serum phosphorus was associated with a greater AF risk: the hazard ratio of AF with a $1-\mathrm{mg} / \mathrm{dl}$ increase in serum phosphorus was 1.13 (95\% confidence interval 1.02 to 1.26 ). No significant interaction was seen by race $(\mathrm{p}=0.88)$ or gender $(\mathrm{p}=0.51)$. The risk of AF was increased in association with greater serum phosphorus in those with an estimated glomerular filtration rate of $\geq 90 \mathrm{ml} / \mathrm{min} / 1.72 \mathrm{~m}^{2}$ but not among those with an estimated glomerular filtration rate of $<90 \mathrm{ml} / \mathrm{min} / 1.72 \mathrm{~m}^{2}$. The total corrected calcium levels were not related to AF risk; however, greater levels of the calcium-phosphorus product were associated with greater AF risk. In conclusion, in the present large population-based study, greater levels of serum phosphorus and the related calcium-phosphorus product were associated with a greater incidence of AF.
\end{abstract}

Atrial fibrillation (AF) is the most common sustained cardiac arrhythmia and is associated with an increased risk of heart failure, stroke, and cardiovascular death. ${ }^{1}$ The established

\footnotetext{
(c) 2013 Elsevier Inc. All rights reserved.

*Corresponding author: Tel: (612) 626-9096; fax: (612) 624-0314. flopez@umn.edu (F.L. Lopez).

Supplementary data

Supplementary data related to this article can be found at doi: http://dx.doi.org/10.1016/j.amjcard.2012.11.045.

Disclosures

The authors have no conflicts of interest to disclose.
} 
risk factors include age, white race, obesity, heart failure, coronary heart disease, left ventricular hypertrophy, hypertension, certain lifestyle factors, ${ }^{2-4}$ and, recently, chronic kidney disease (CKD). ${ }^{5}$ Increased phosphatemia because of impaired phosphorus excretion with decreased kidney function could help explain the relation between AF and CKD if phosphorus is an independent predictor of AF. Phosphorus is essential for numerous biologic functions, including cellular signal transduction, mineral metabolism, and energy exchange. ${ }^{6}$ Elevated phosphorus levels have been independently linked with calcification of the aorta and coronary arteries, increased arterial stiffness, elevated left ventricular mass, carotid atherosclerosis, ${ }^{7-13}$ and increased cardiovascular morbidity and mortality in subjects with and without CKD. ${ }^{6,11,14-18}$ The present study estimated the association of serum phosphorus levels with the incidence of AF in the Atherosclerosis Risk In Communities (ARIC) study cohort. We also assessed whether the related serum calcium and calciumphosphorus product were associated with AF risk.

\section{Methods}

The ARIC study was a biracial, prospective cohort study of cardiovascular disease and atherosclerosis risk factors. ${ }^{19}$ The participants at baseline (1987 to 1989) included 15,792 men and women aged 45 to 64 years, recruited from 4 communities in the United States (Washington County, Maryland; northwest suburbs of Minneapolis, Minnesota; Jackson, Mississippi; and Forsyth County, North Carolina). Additional details on the ARIC study can be found in the online supplement. The institutional review board at each participating center approved the present study, and all study participants provided written informed consent.

The AF diagnoses were ascertained by 3 different sources in the ARIC study: electrocardiograms performed at study visits, hospital discharge codes, and death certificates. ${ }^{2}$ Additional details of $\mathrm{AF}$ ascertainment can be found in the online supplement.

In the present analysis, the incidence date of $\mathrm{AF}$ was defined as the date for the first electrocardiogram showing AF, the first hospital discharge coded as AF, or when AF was listed as a cause of death, whichever occurred earlier.

Blood samples were collected at each visit after a fast of $\geq 8$ hours and sent to the ARIC Central Lipid Laboratory. The phosphorus, calcium, and creatinine levels were measured in frozen serum samples, using methods based on ammonium molybdate, $o$-cresolphthalein complexone, and modified kinetic Jaffe-picric acid, respectively. ${ }^{19,20}$ The coefficient of variation was $2.4 \%$ for phosphorus, $1.9 \%$ for calcium, and $3.7 \%$ for creatinine. Detailed procedures on baseline covariate measurements have been previously published ${ }^{19}$ and are also available in the online supplement.

Of the 15,792 participants who attended visit 1 in the ARIC study, we excluded those who were of a racial group other than white or black and nonwhites in the Minneapolis and Washington County field centers $(n=103)$, those with prevalent AF at visit $1(n=37)$, lowquality or missing electrocardiograms $(n=242)$, those missing phosphorus levels $(n=124)$, those with nonfasting blood samples $(n=485)$, those missing covariates $(n=108)$, and those with an estimated glomerular filtration rate $(\mathrm{eGFR})<15 \mathrm{ml} / \mathrm{min} / 1.73 \mathrm{~m}^{2}(\mathrm{n}=18)$. The final sample included 14,675 participants ( $25 \%$ black, $45 \%$ men).

We estimated the association between the baseline serum phosphorus and calcium levels and the incidence of AF using Cox proportional hazards models, with the interval to AF as the main outcome variable. Initially, we explored the association between phosphorus and calcium and AF risk using restricted cubic splines. Next, we categorized the serum phosphorus and calcium levels and the calcium-phosphorus product by quintiles for the 
categorical analysis. $\mathrm{p}$ Values for trend were calculated across the quintile categories using the quintile term. The follow-up period was defined as the interval elapsed between baseline and the date of AF incidence, death, lost to follow-up, or December 31, 2008, whichever came earlier. Models for the association of the phosphorus levels with AF risk were adjusted for the baseline covariates, including age, gender, race, study site, education (less than completed high school, high school diploma, at least some college), height (continuous), smoking (never, former, current), alcohol drinking (never, former, current), body mass index (continuous), diabetes (dichotomous), serum calcium (adjusted for albumin), systolic blood pressure (continuous), diastolic blood pressure (continuous), use of antihypertensive medications, eGFR (modeled as a spline), prevalent stroke, prevalent heart failure, and prevalent coronary heart disease. An additional model was performed, adjusting for incident heart failure and incident coronary heart disease as timevarying covariates. Models considering calcium as the main exposure included the same covariates, except for adjusting for phosphorus instead of calcium.

Because both excess phosphorus and an increased risk of AF have been independently linked with CKD, we also examined the association of phosphorus and AF stratified by the eGFR. Effect measure modification on the multiplicative scale was formally tested in the multivariate model, including an interaction term between gender, race, and eGFR categories and the phosphorus levels. The proportional hazards assumption was tested using time interaction terms and inspection of log negative log survival curves. All statistical analyses were performed with SAS, version 9.2 (SAS Institute, Cary, North Carolina).

\section{Results}

Selected characteristics for the ARIC participants by quintile of serum phosphorus level are listed in Table 1. Women, blacks, current smokers, and those with prevalent heart failure were more likely to have higher phosphorus levels. The mean \pm SD of serum phosphorus was $3.4 \pm 0.5 \mathrm{mg} / \mathrm{dl}$ (interquartile range 3.1-3.7). The mean $\pm \mathrm{SD}$ of calcium and the calcium-phosphorus product was $9.9 \pm 0.4 \mathrm{mg} / \mathrm{dl}$ and $33.9 \pm 5.2$, respectively. The correlation coefficient between phosphorus and calcium was 0.16 and between phosphorus and the calcium-phosphorus product was 0.96 .

During a median follow-up of 19.7 years, we identified 1,656 incident AF cases. The association between the serum phosphorus level and incident AF was examined using cubic splines, showing a slight $\mathrm{J}$-shaped or reversed L-shaped association (Figure 1), with a nonsignificant phosphorus quadratic term $(\mathrm{p}=0.32)$. The multivariate adjusted hazard ratio of AF associated with 1-mg/dl higher serum phosphorus level was 1.13 (95\% confidence interval 1.02 to 1.26). Categorizing serum phosphorus in quintiles, a $20 \%$ greater risk of $\mathrm{AF}$ was observed in the top quintiles compared to the lowest quintile, even after adjustment for potential confounders (Table 2). The results were slightly attenuated in the uppermost quintile with additional adjustment for time-varying heart failure and coronary heart disease. The results did not differ with additional adjustment for incident hypertension, diuretic use, vitamin $\mathrm{D}$ intake, or after adjustment for eGFR at visit 4 (data not shown).

After adjustment for potential confounders, the calcium levels were not associated with a greater risk of $\mathrm{AF}$, either as a continuous variable (hazard ratio $1.10,95 \%$ confidence interval 0.97 to 1.24 , for a $1-\mathrm{mg} / \mathrm{dl}$ difference) or when categorized in quintiles (Table 2).

The association between the calcium-phosphorus product and AF risk was similar to that for serum phosphorus alone (Table 2). The results were only slightly attenuated after additional adjustment for incident heart failure and coronary heart disease and remained significant. 
No significant interaction was seen in the association between phosphorus and AF by race ( $\mathrm{p}$ $=0.88)$ or gender $(\mathrm{p}=0.51)$. Associations between serum phosphorus and AF by gender are reported in Table E1. Owing to the narrow interquartile range, we performed a sensitivity analysis, excluding those with phosphorus levels lower than the 1st percentile and greater than the 99th percentile to explore the influence of extreme values. The significance of the association between phosphorus and AF did not change. The interaction between phosphorus and AF by eGFR category was not significant $(\mathrm{p}=0.18)$. There was an increased risk of AF associated with higher serum phosphorus levels in those with an eGFR $\searrow 90 \mathrm{ml} / \mathrm{min} / 1.73 \mathrm{~m}^{2}$ but not among those with an eGFR $<90 \mathrm{ml} / \mathrm{min} / 1.73 \mathrm{~m}^{2}$ (Table 3). The association of serum phosphorus with incident AF by kidney function using cubic splines showed slight $\mathrm{J}$-shaped associations in those with an eGFR $>90$ or $<60 \mathrm{ml} / \mathrm{min} / 1.73 \mathrm{~m}^{2}$, and no association in those with an eGFR of 60 to $90 \mathrm{ml} / \mathrm{min} / 1.73 \mathrm{~m}^{2}$ (Figure 2).

\section{Discussion}

In the present population-based prospective study, greater serum phosphorus levels and the related calciumphosphorus product were associated with an increased risk of incident AF. Phosphorus was associated with a greater AF risk in those with normal kidney function (eGFR $\searrow 00 \mathrm{ml} / \mathrm{min} / 1.73 \mathrm{~m}^{2}$ ); thus, the effect of phosphorus is not driven solely by poor kidney function. This association was independent of lifestyle factors, clinical factors, and cardiovascular disease and was similar in terms of gender and race. In our analysis, the association between phosphorus and $\mathrm{AF}$ was similar in direction and magnitude to the associations seen between phosphorus levels and increased risks of cardiovascular disease and mortality in patients with CKD or coronary disease and in the general population. $6,14,16-18$

Several potential mechanisms might explain the increased risk of AF with greater phosphorus levels. First, excess phosphorus levels have been linked to calcification of the aorta and coronary arteries, ${ }^{7,8}$ thereby increasing vascular calcification and smooth muscle proliferation and directly promoting vascular injury, which could lead to greater AF risk. Second, high phosphorus levels are known to inhibit 1,25-dihydroxyvitamin D synthesis, ${ }^{21}$ and it has been hypothesized that lower 1,25-dihydroxyvitamin D levels also increase coronary calcification and decrease cardiac contractility, ${ }^{22,23}$ possibly leading to increased an risk of AF. Third, excess phosphorus has been associated with secondary hyperparathyroidism, which involves increased circulating levels of parathyroid hormone (PTH). High PTH levels might be proinflammatory, leading to an increased risk of AF. In a recent cross-sectional study, PTH was elevated in patients with AF compared to controls in sinus rhythm. ${ }^{24}$ However, the associations of phosphorus with death or cardiovascular outcomes were not affected after adjustments for $\mathrm{PTH}^{14}$ or the inflammatory marker, highsensitivity C-reactive protein, in other populations. ${ }^{17}$ Other mechanisms that could mediate the association between serum phosphorus and AF risk include an increased left ventricular mass and greater risk of heart failure in those with higher phosphorus levels. ${ }^{11}$ Finally, fibroblast growth factor 23, a phosphate-regulating hormone that reflects serum phosphorus levels, has been associated with left ventricular dysfunction and the prevalence of AF in patients undergoing coronary angiography. ${ }^{25}$

No clear association was found between the calcium level and AF risk. Therefore, the observed association between the calcium-phosphorus product and AF was probably attributable to serum phosphorus. These results are consistent with those of previous studies of other cardiovascular outcomes. ${ }^{14,15,17,18}$

In the present analysis, we found that serum phosphorus was associated with greater AF risk only among those with normal kidney function, as measured by the eGFR, but not among 
those with reduced eGFR, although the reduced eGFR groups had fewer outcomes and low power. The phosphorus levels correlated with kidney function, and because it does not appear that elevated phosphorus is on the pathway between CKD and AF, we can only speculate about the reasons for this potential interaction, given that in ARIC, participants with a lower eGFR had a greater risk of $\mathrm{AF}^{5}$ If other mechanisms different from serum phosphorus are associated with increased AF risk in those with lower eGFR, higher phosphorus levels might not result in additional risk in this group. Our observation could be a chance finding, and future studies should try to replicate it.

Our study had major strengths, including a large number of incident AF cases, which provided adequate power to identify moderate associations, the community-based biracial sample, which increases the generalizability of our results, and the availability of an extensive list of measured covariates, which allowed adequate adjustment for most confounders. Still, some study limitations should be noted. Asymptomatic AF and AF managed exclusively in an outpatient facility could not be identified, because the large majority of our incident AF cases were ascertained from the hospitalization discharge records. We have found, however, adequate validity of these codes for the identification of $\mathrm{AF}$ events. ${ }^{2}$ The strength of the association between phosphorus and AF was modest, and residual confounding, if present, could explain the association. However, we adjusted for most known risk factors that were measured well in the ARIC study, making this less likely. Misclassification of the exposure could also occur because of within-person variability in serum phosphorus levels. Finally, the ARIC study did not contain information on thyroid profiles or PTH, which could potentially be confounders in our study.

\section{Supplementary Material}

Refer to Web version on PubMed Central for supplementary material.

\section{Acknowledgments}

We thank the staff and participants of the ARIC study for their important contributions.

The Atherosclerosis Risk In Communities Study was performed as a collaborative study supported by contracts HHSN268201100005C, HHSN268201100006C, HHSN268201100007C, HHSN268201100008C, HHSN268201100009C, HHSN268201100010C, HHSN268201100011C, and HHSN268201100012C from the National Heart, Lung, and Blood Institute (Bethesda, Maryland). This study was additionally supported by grant RC1-HL099452 from the National Heart, Lung, and Blood Institute (Bethesda, Maryland) and grant 09SDG2280087 from the American Heart Association (Dallas, Texas).

\section{References}

1. Benjamin EJ, Levy D, Vaziri SM, D’Agostino RB, Belanger AJ, Wolf PA. Independent risk factors for atrial fibrillation in a population-based cohort: the Framingham Heart Study. JAMA. 1994; 271:840-844. [PubMed: 8114238]

2. Alonso A, Agarwal SK, Soliman EZ, Ambrose M, Chamberlain AM, Prineas RJ, Folsom AR. Incidence of atrial fibrillation in whites and African-Americans: the Atherosclerosis Risk In Communities (ARIC) study. Am Heart J. 2009; 158:111-117. [PubMed: 19540400]

3. Sanoski CA. Prevalence, pathogenesis, and impact of atrial fibrillation. Am J Health Syst Pharm. 2010; 67:S11-S16. [PubMed: 20410531]

4. Wang TJ, Parise H, Levy D, D’ Agostino RB Sr, Wolf PA, Vasan RS, Benjamin EJ. Obesity and the risk of new-onset atrial fibrillation. JAMA. 2004; 292:2471-2477. [PubMed: 15562125]

5. Alonso A, Lopez FL, Matsushita K, Loehr LR, Agarwal SK, Chen LY, Soliman EZ, Astor BC, Coresh J. Chronic kidney disease is associated with the incidence of atrial fibrillation: the Atherosclerosis Risk In Communities (ARIC) study. Circulation. 2011; 123:2946-2953. [PubMed: 21646496] 
6. Tonelli M, Sacks F, Pfeffer M, Gao Z, Curhan G. Cholesterol and Recurrent Events Trial Investigators. Relation between serum phosphate level and cardiovascular event rate in people with coronary disease. Circulation. 2005; 112:2627-2633. [PubMed: 16246962]

7. Raggi P, Boulay A, Chasan-Taber S, Amin N, Dillon M, Burke SK, Chertow GM. Cardiac calcification in adult hemodialysis patients: a link between end-stage renal disease and cardiovascular disease? J Am Coll Cardiol. 2002; 39:695-701. [PubMed: 11849871]

8. Goodman WG, Goldin J, Kuizon BD, Yoon C, Gales B, Sider D, Wang Y, Chung J, Emerick A, Greaser L, Elashoff RM, Salusky IB. Coronary- artery calcification in young adults with end-stage renal disease who are undergoing dialysis. N Engl J Med. 2000; 342:1478-1483. [PubMed: 10816185]

9. Foley RN, Collins AJ, Herzog CA, Ishani A, Kalra PA. Serum phosphorus levels associate with coronary atherosclerosis in young adults. J Am Soc Nephrol. 2009; 20:397-404. [PubMed: 18987306]

10. Ix JH, De Boer IH, Peralta CA, Adeney KL, Duprez DA, Jenny NS, Siscovick DS, Kestenbaum BR. Serum phosphorus concentrations and arterial stiffness among individuals with normal kidney function to moderate kidney disease in MESA. Clin J Am Soc Nephrol. 2009; 4:609-615. [PubMed: 19211667]

11. Dhingra R, Gona P, Benjamin EJ, Wang TJ, Aragam J, D’ Agostino RB Sr, Kannel WB, Vasan RS. Relations of serum phosphorus levels to echocardiographic left ventricular mass and incidence of heart failure in the community. Eur J Heart Fail. 2010; 12:812-818. [PubMed: 20675668]

12. Saab G, Whooley MA, Schiller NB, Ix JH. Association of serum phosphorus with left ventricular mass in men and women with stable cardiovascular disease: data from the Heart and Soul Study. Am J Kidney Dis. 2010; 56:496-505. [PubMed: 20580478]

13. Onufrak SJ, Bellasi A, Shaw LJ, Herzog CA, Cardarelli F, Wilson PW, Vaccarino V, Raggi P. Phosphorus levels are associated with subclinical atherosclerosis in the general population. Atherosclerosis. 2008; 199:424-431. [PubMed: 18093595]

14. Block GA, Hulbert-Shearon TE, Levin NW, Port FK. Association of serum phosphorus and calcium $\times$ phosphate product with mortality risk in chronic hemodialysis patients: a national study. Am J Kidney Dis. 1998; 31:607-617. [PubMed: 9531176]

15. Ganesh SK, Stack AG, Levin NW, Hulbert-Shearon T, Port FK. Association of elevated serum $\mathrm{PO}(4), \mathrm{Ca} \times \mathrm{PO}(4)$ product, and parathyroid hormone with cardiac mortality risk in chronic hemodialysis patients. J Am Soc Nephrol. 2001; 12:2131-2138. [PubMed: 11562412]

16. Kestenbaum B, Sampson JN, Rudser KD, Patterson DJ, Seliger SL, Young B, Sherrard DJ, Andress DL. Serum phosphate levels and mortality risk among people with chronic kidney disease. J Am Soc Nephrol. 2005; 16:520-528. [PubMed: 15615819]

17. Dhingra R, Sullivan LM, Fox CS, Wang TJ, D’ Agostino RB Sr, Gaziano JM, Vasan RS. Relations of serum phosphorus and calcium levels to the incidence of cardiovascular disease in the community. Arch Intern Med. 2007; 167:879-885. [PubMed: 17502528]

18. Foley RN, Collins AJ, Ishani A, Kalra PA. Calcium-phosphate levels and cardiovascular disease in community-dwelling adults: the Atherosclerosis Risk In Communities (ARIC) study. Am Heart J. 2008; 156:556-563. [PubMed: 18760141]

19. The ARIC Investigators. The Atherosclerosis Risk In Communities (ARIC) study: design and objectives. Am J Epidemiol. 1989; 129:687-702. [PubMed: 2646917]

20. National Heart, Lung and Blood Institute. Clinical Chemistry Determinations. Bethesda: National Heart, Lung and Blood Institute; 1987. Atherosclerosis Risk In Communities (ARIC) Study: Operations Manual No 10; p. 1-76.

21. Portale AA, Halloran BP, Morris RC Jr. Physiologic regulation of the serum concentration of 1,25dihydroxyvitamin D by phosphorus in normal men. J Clin Invest. 1989; 83:1494-1499. [PubMed: 2708521]

22. Watson KE, Abrolat ML, Malone LL, Hoeg JM, Doherty T, Detrano R, Demer LL. Active serum vitamin D levels are inversely correlated with coronary calcification. Circulation. 1997; 96:17551760. [PubMed: 9323058] 
23. Zittermann A, Schleithoff SS, Tenderich G, Berthold HK, Korfer R, Stehle P. Low vitamin D status: a contributing factor in the pathogenesis of congestive heart failure? J Am Coll Cardiol. 2003; 41:105-112. [PubMed: 12570952]

24. Rienstra M, Lubitz SA, Zhang ML, Cooper RR, Ellinor PT. Elevation of parathyroid hormone levels in atrial fibrillation. J Am Coll Cardiol. 2011; 57:2542-2543. [PubMed: 21679857]

25. Seiler S, Cremers B, Rebling NM, Hornof F, Jeken J, Kersting S, Steimle C, Ege P, Fehrenz M, Rogacev KS, Scheller B, Bohm M, Fliser D, Heine GH. The phosphaton in fibroblast growth factor 23 links calcium-phosphate metabolism with left-ventricular dysfunction and atrial fibrillation. Eur Heart J. 2011; 32:2688-2696. [PubMed: 21733911] 


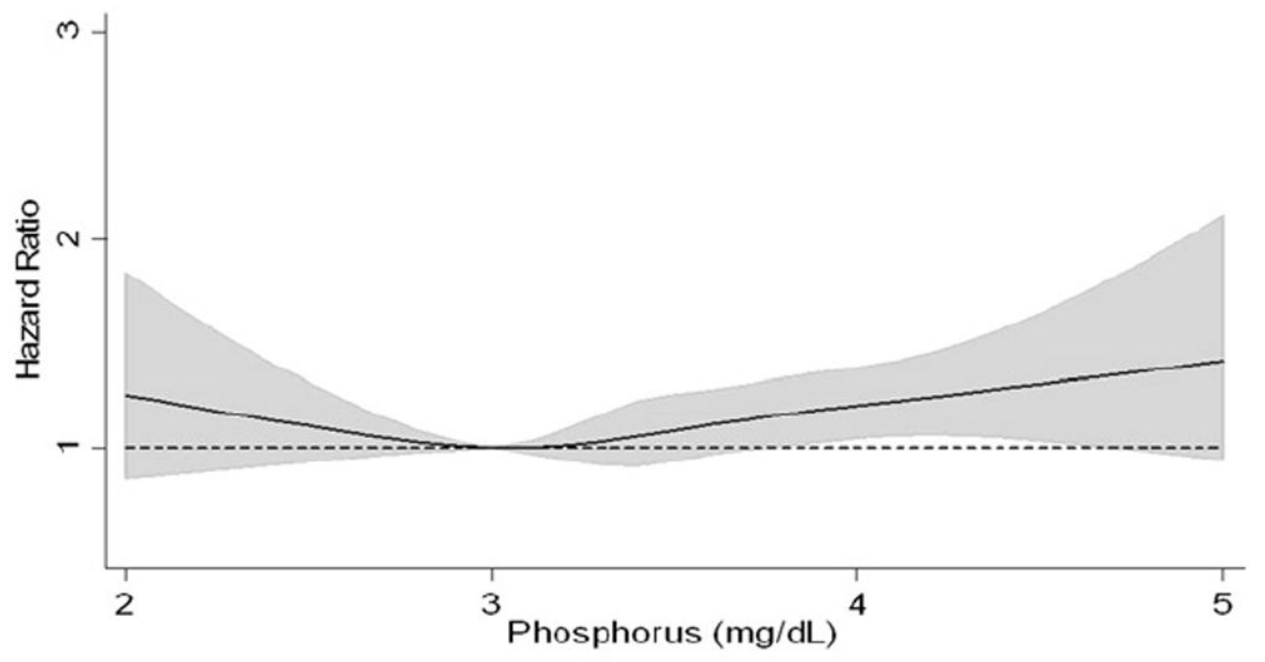

Relative hazard

$95 \% \mathrm{Cl} \quad----$ Reference line $=1$

Figure 1.

Association between serum phosphorus levels, modeled as restricted cubic spline, and AF (ARIC 1987 to 2008). Model adjusted for age, gender, and race. Black line represents hazard ratio; gray shaded area, 95\% confidence interval. 

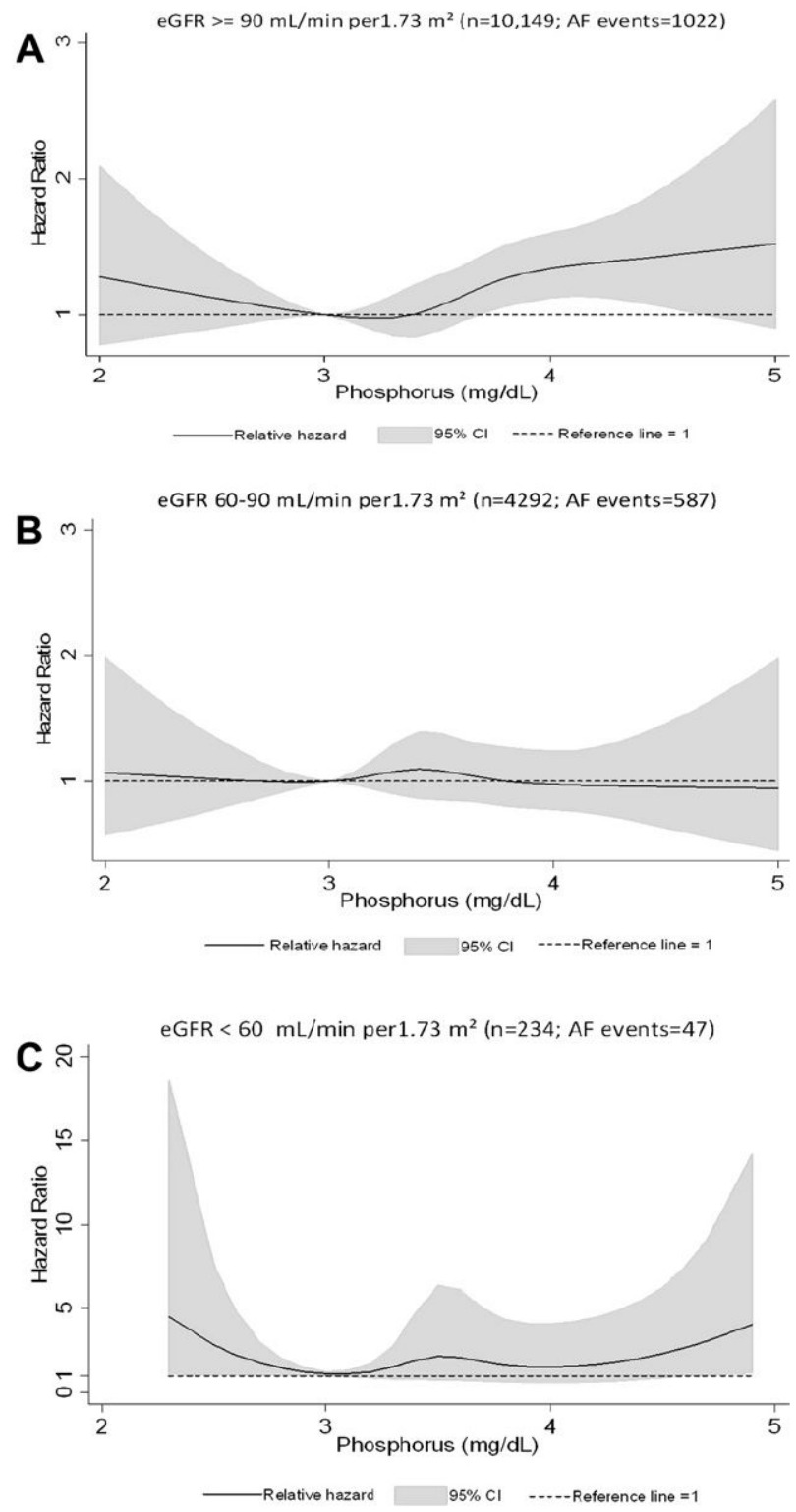

Figure 2.

Association between serum phosphorus levels, modeled as restricted cubic spline, and AF and stratified by eGFR ( $>90,60$ to $90,<60 \mathrm{ml} / \mathrm{min} / 1.73 \mathrm{~m}^{2}$ ), ARIC 1987 to 2008 . Models adjusted for age, gender, and race. Black line represents hazard ratio; gray shaded area, $95 \%$ confidence interval. (A) eGFR $290 \mathrm{ml} / \mathrm{min} / 1.73 \mathrm{~m}^{2}(\mathrm{n}=10,149 ;$ AF events $=1,022),(B)$ eGFR 60 to $90 \mathrm{ml} / \mathrm{min} / 1.73 \mathrm{~m}^{2}(\mathrm{n}=4,292 ;$ AF events $=587),(C)$ eGFR $<60 \mathrm{ml} / \mathrm{min} / 1.73$ $\mathrm{m}^{2}(\mathrm{n}=234 ; \mathrm{AF}$ events $=47)$. 


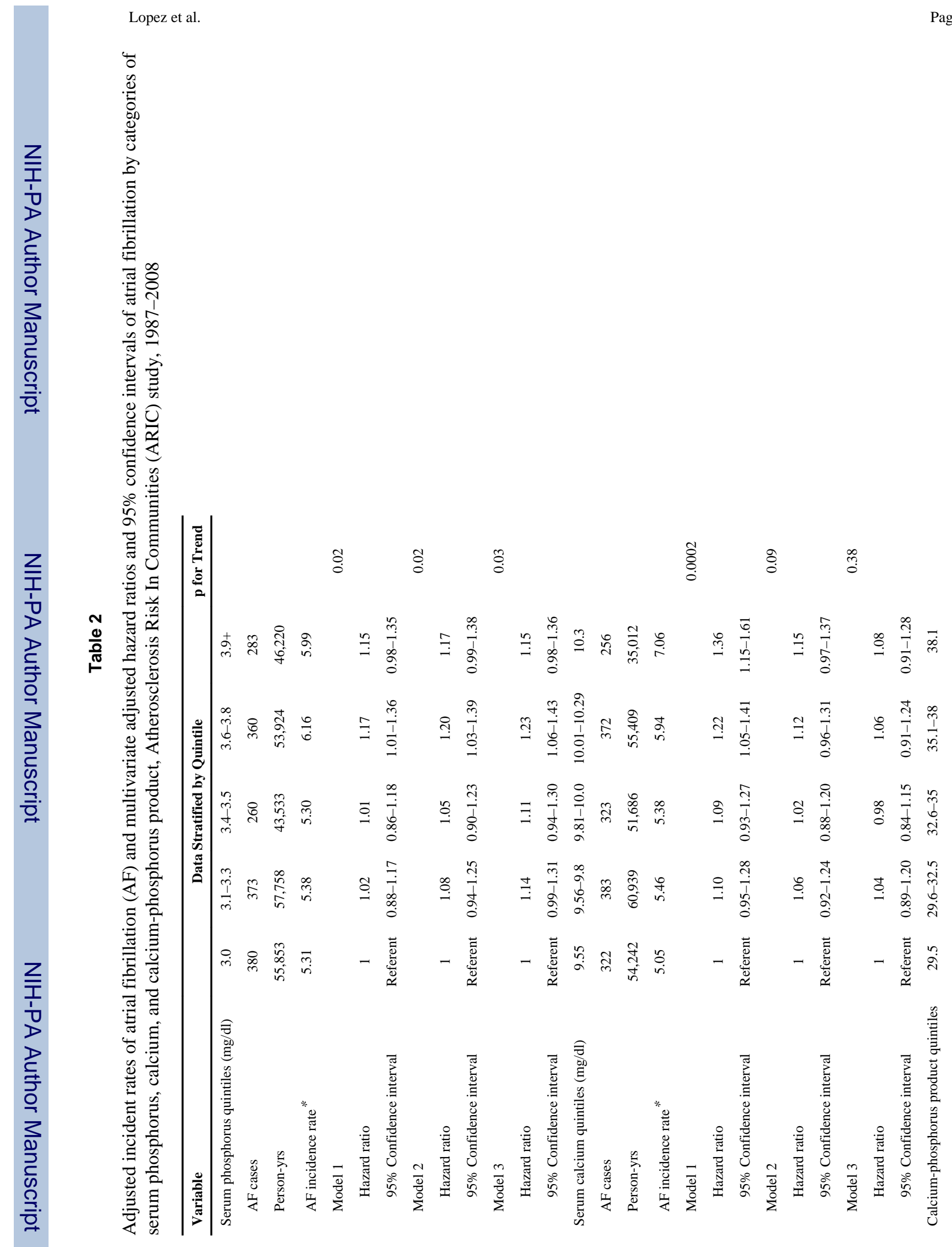




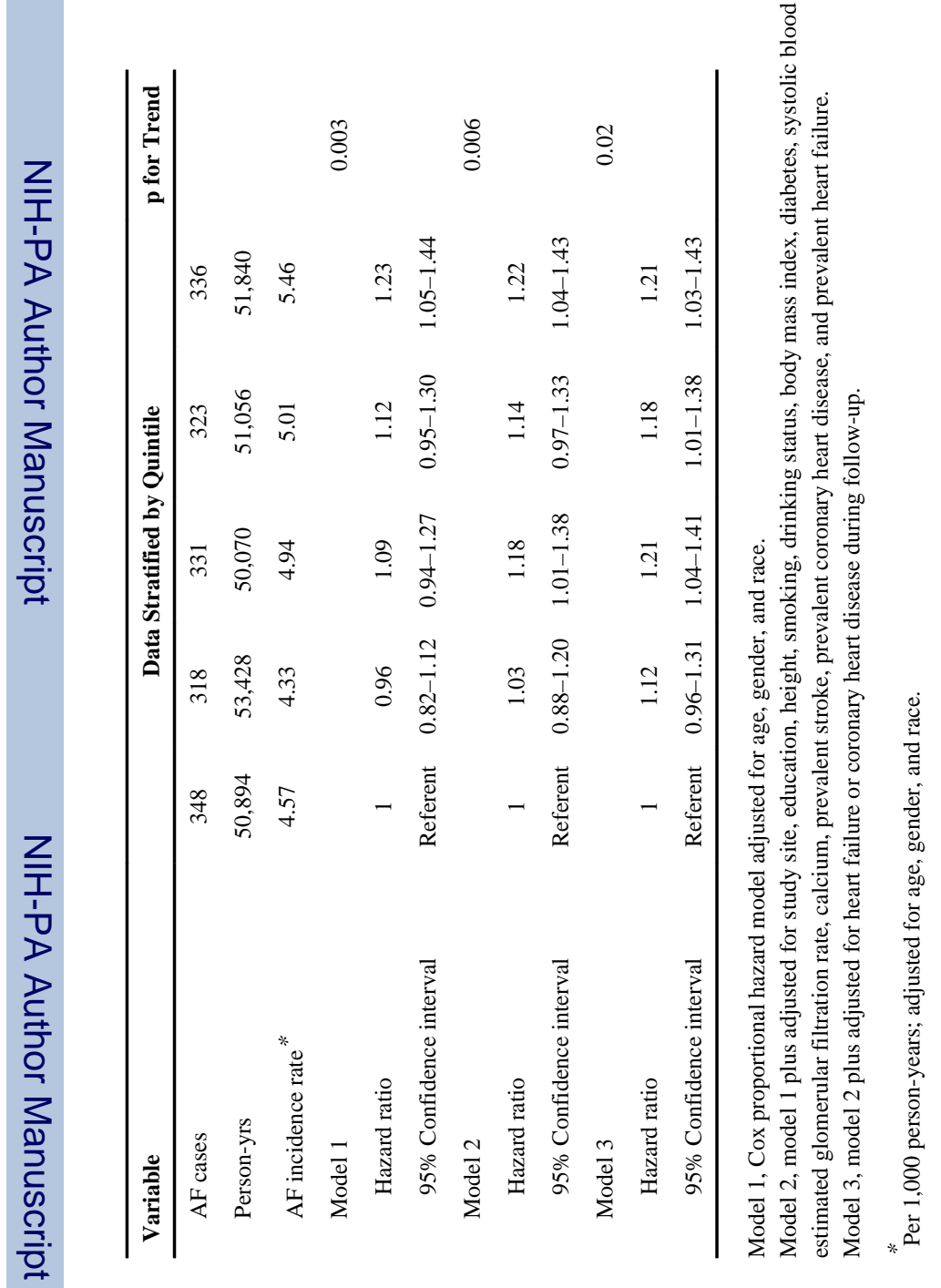




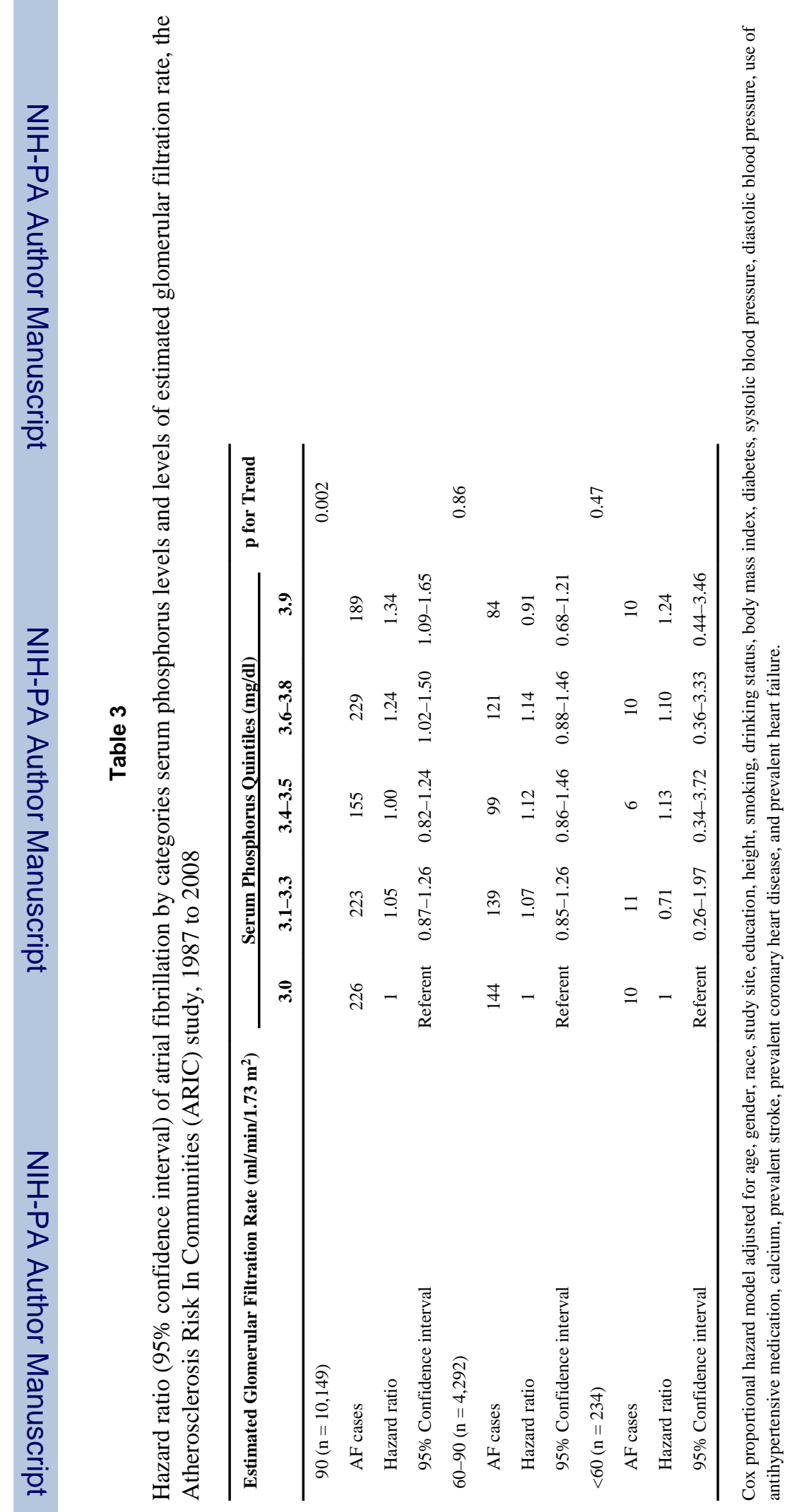

